\title{
Раскрытие энергетической щели в области точки Дирака при осаждении кобальта на поверхность (0001) топологического изолятора $\mathrm{BiSbTeSe}_{2}$
}

\author{
(C) А.К. Кавеев ${ }^{1}$, А.Г Банщиков ${ }^{1}$, А.Н Терпицкий ${ }^{1}$, В.А Голяшов ${ }^{2}$, О.Е Терещенко ${ }^{2}$, K.A Kох ${ }^{3}$, \\ Д.А Естюнин ${ }^{4}$, А.М Шикин ${ }^{4}$ \\ ${ }^{1}$ Физико-технический институт им. А.Ф. Иоффре Российской академии наук, \\ 194021 Санкт-Петербург, Россия \\ ${ }^{2}$ Институт фризики полупроводников им. А.В. Ржанова Сибирского отделения Российской академии наук, \\ 630090 Новосибирск, Россия \\ ${ }^{3}$ Институт геологии и минералогии им. В.С. Соболева Сибирского отделения Российской академии наук, \\ 630090 Новосибирск, Россия \\ ${ }^{4}$ Санкт-Петербургский государственный университет, \\ 199034 Санкт-Петербург, Россия \\ E-mail: kaveev@mail.ioffe.ru
}

Поступила в Редакцию 15 апреля 2020 г.

В окончательной редакции 21 апреля 2020 г.

Принята к публикации 21 апреля 2020 г.

Впервые показано, что субнанометровые покрытия Сo, нанесенные методом молекулярно-лучевой эпитаксии на поверхность (0001) топологического изолятора BiSbTeSe 2 при температуре $330^{\circ} \mathrm{C}$, открывают энергетическую щель в спектре топологических поверхностных состояний в области точки Дирака, со смещением положения точки Дирака, вызванным предварительным осаждением адсорбата при комнатной температуре. Ширина щели составляет $21 \pm 6$ мэВ. Температурно-зависимые измерения в диапазоне $15-150 \mathrm{~K}$ не показали изменения ширины энергетической щели.

Ключевые слова: топологические изоляторы, спинтроника, энергетическая щель в точке Дирака, легирование.

DOI: $10.21883 /$ FTP.2020.09.49821.13

\section{1. Введение}

В настоящее время выраженный научный интерес в физике твердого тела и физике полупроводников привлекают топологические изоляторы (ТИ). Поверхностные состояния этих материалов обладают экзотическими свойствами и топологически защищены симметрией к обращению времени [1,2]. Эти материалы имеют уникальную электронную и спиновую структуру, заключающуюся в наличии запрещенной зоны в объеме материала и наличии дираковского конуса спин-поляризованных проводящих поверхностных состояний, формируемых вследствие сильного спин-орбитального взаимодействия. Нанесение сверхтонких ферромагнитных (ФМ) покрытий на поверхность ТИ привлекательно с точки зрения контроля проводящих свойств поверхностных состояний: возможно снятие топологической защищенности за счет эффекта близости, заключающегося во влиянии магнитного момента ферромагнетика на спин-поляризованные поверхностные состояния ТИ [3]. Это снятие инверсии по времени должно приводить к снятию топологической защищенности и открытию энергетической щели в области точки Дирака. Обратный эффект заключается в возможном влиянии тока по спин-поляризованным состояниям на магнитный момент ферромагнитного покрытия $[4,5]$. С точки зрения упомянутого применения эпитаксиальных систем ФМ/ТИ важно изучить измене- ние электронных, структурных и интерфейсных свойств ТИ при осаждении ферромагнитного материала. Существует несколько работ, связанных с экспериментальными исследованиями контакта ФМ и ТИ в различных системах, например, с использованием $\mathrm{Bi}_{2} \mathrm{Se}_{3}[6]$ или $\mathrm{Sb}_{2} \mathrm{Te}_{3}[7,8]$ в качестве ТИ. В настоящей работе были проведены исследования трансформации структуры $\mathrm{BiSbTeSe}_{2}$ и электронных состояний при осаждении Co.

\section{2. Экспериментальные методики}

Подложки четверного твердого раствора $\mathrm{BiSbTeSe}_{2}$ были получены методом Бриджмена. Чистая поверхность BiSbTeSe 2 получалась путем скалывания в сверхвысоком вакууме $\left(10^{-9}\right.$ мбар). Нанесение ферромагнитных слоев Со осуществлялось в диапазоне температур от комнатной до $340^{\circ} \mathrm{C}$ методом молекулярнолучевой эпитаксии (МЛЭ) путем разогрева материала электронным ударом. Эффективная толщина наносимых покрытий составляла $0-2 \AA$. Исследование электронной структуры поверхностных состояний проводилось методом лазерной фотоэлектронной спектроскопии [9] с угловым разрешением (ЛФЭСУР) на установке центра синхротронных исследований HiSOR (г. Хиросима, Япония) на основе титан-сапфирового лазера (Tsunami, Spectra Physics) с длиной волны 191-210 нм и мощностью $0.1-0.7$ мВт. Для измерения угловой зависимости 

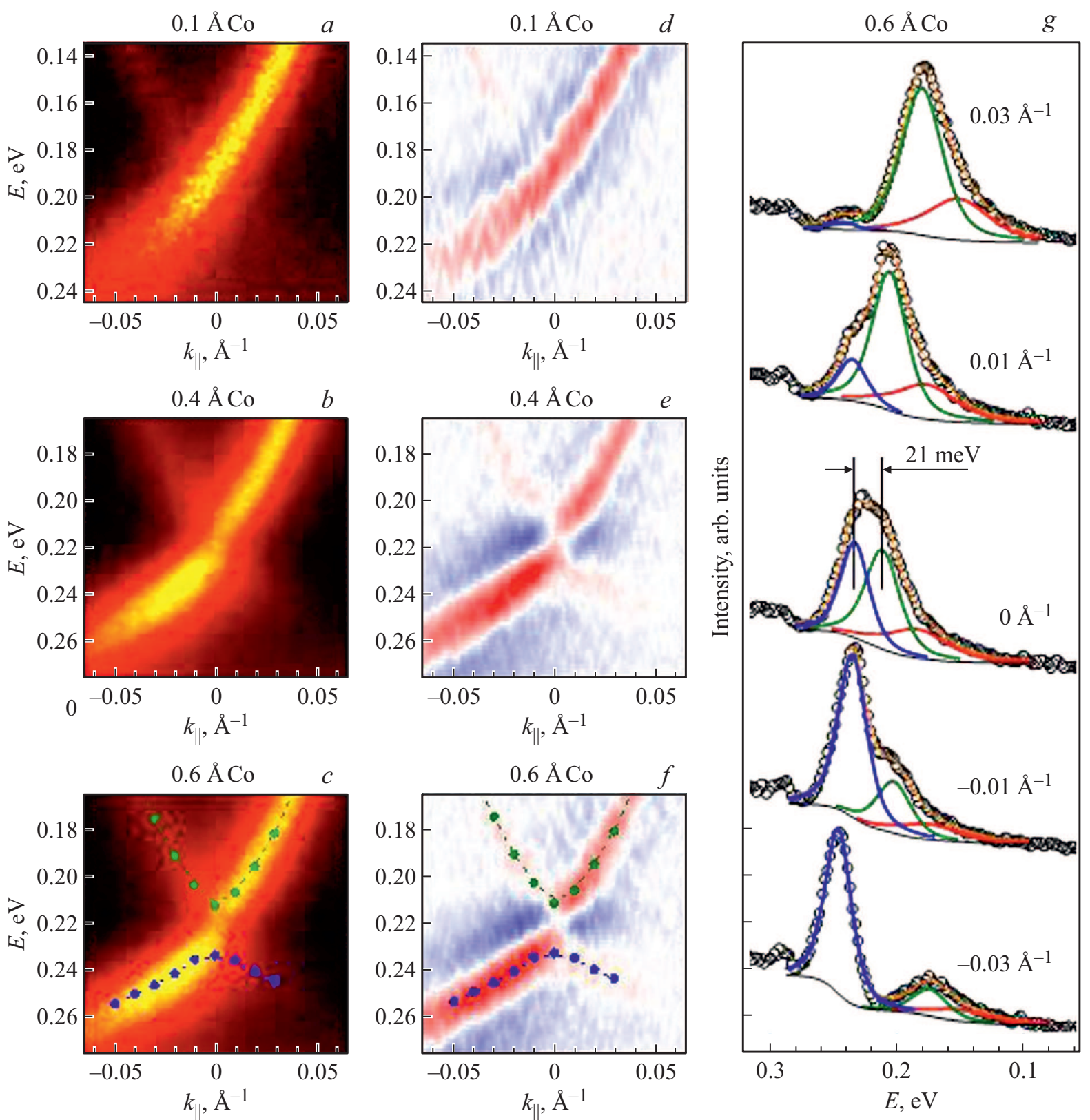

Рис. 1. Спектры ЛФЭСУР топологических поверхностных состояний $\mathrm{BiSbTeSe}_{2}$ вблизи точки Дирака (направление $\left.Г-K\right)$ для образцов с $0.1(a), 0.4(b)$ и $0.6 \AA(c)$ Со с их вторыми производными $d^{2} I / d E^{2}(d-f)$ соответственно. $g$ - профили интенсивности $\mathrm{EDC}$ для разных $k_{\|}$значений спектра на этом рисунке $(0.6 \AA \mathrm{Co})$. Данные были подогнаны с использованием двух профилей Войта для нижнего (синяя кривая) и верхнего (зеленая кривая) состояний конуса Дирака и фона Ширли, третий профиль Войта (красная кривая) использовался для учета асимметричного хвоста с более низкой энергией связи. Полученные энергетические положения подобранных пиков изображены в виде точек и пунктирных линий в $(c)$ и $(f)$.

энергии фотоэлектронов использовался спектрометр с угловым разрешением по двум координатам. Метод ЛФЭСУР является мощным инструментом для изучения закона дисперсии и особенностей структуры поверхностных состояний. Использование высокоэнергетического лазерного излучения с внешней фокусировкой позволяет достичь уникально высокого ( 5-10 микрон) пространственного разрешения по поверхности образца по сравнению с синхротронным излучением, обычно используемым в системах ФЭСУР (пространственное разрешение 100-1000 микрон). Для поиска Г-точки выполнялось подробное картографирование обратного пространства $\left(k_{x}, k_{y}\right)$ вокруг данной точки.

\section{3. Полученные результаты и обсуждение}

Поверхность $\mathrm{BiSbTeSe}_{2}(0001)$ подготавливалась путем скола в сверхвысоком вакууме ( $10^{-9}$ мБар). 


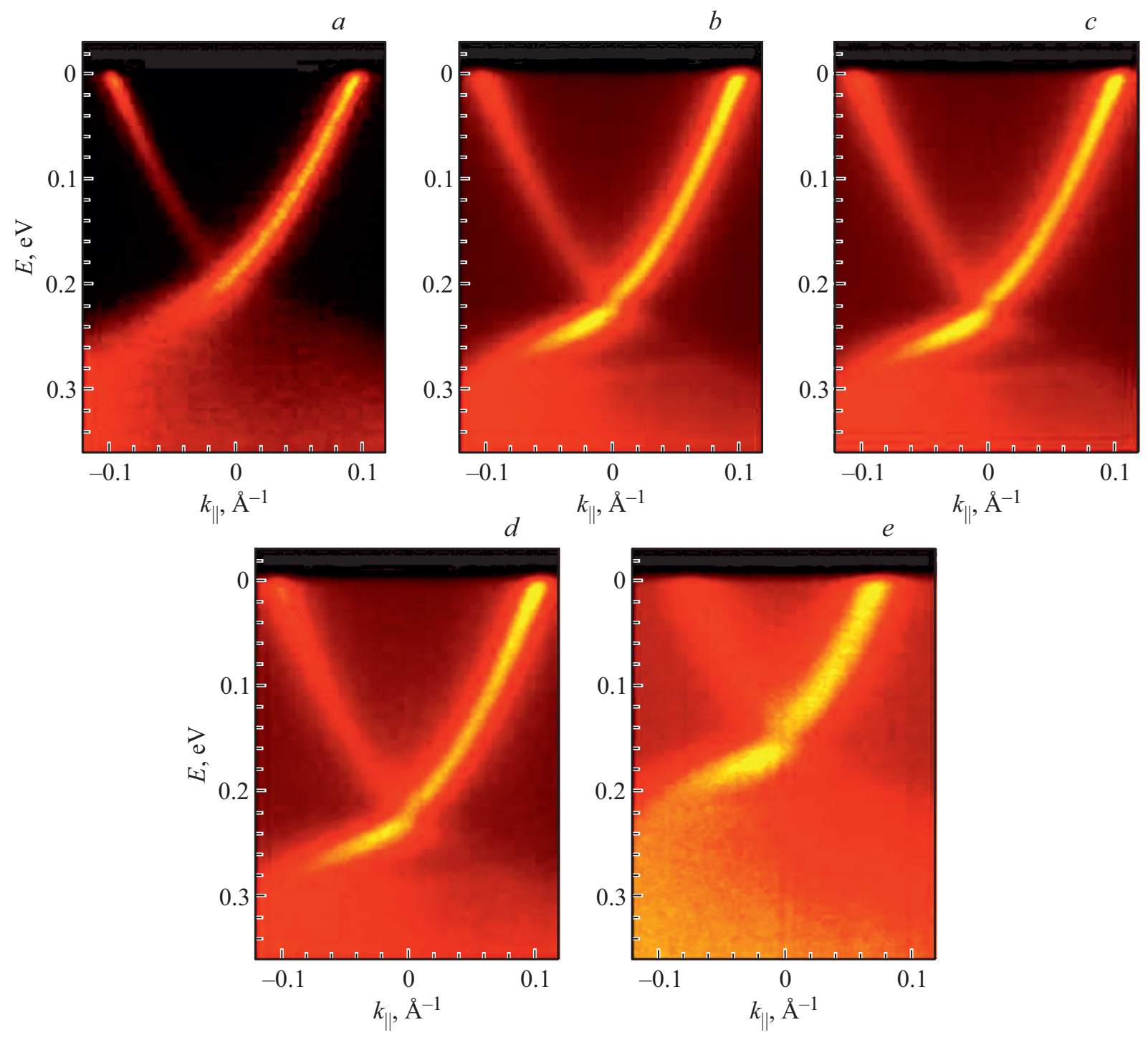

Рис. 2. $a-d-$ изменения в электронной структуре поверхности $\mathrm{BiSbTeSe}_{2}$ при последовательном осаждении $0.1(a), 0.4(b)$, $0.6(c), 1 \AA(d)$ Со и $2 \AA(e)$ Со при температуре подложки $300^{\circ}$ С. Энергетическая щель в области точки Дирака наблюдается во всех указанных случаях.

Методом ЛФЭСУР показано, что чистая поверхность $\mathrm{BiSbTeSe}_{2}$ демонстрирует наличие поверхностных состояний с линейным законом дисперсии дираковского типа, при этом уровень Ферми находится в запрещенной зоне и пересекает поверхностные состояния в точке Дирака. При такой структуре поверхностных состояний наблюдение ее изменений в области точки Дирака методом ЛФЭСУР невозможно. Однако было установлено, что нанесение сверхмалых количеств адсорбата $(\sim 0.1 \AA)$ при комнатной температуре приводит к изгибу зон вниз (электронное легирование) со смещением точки Дирака ниже уровня Ферми. В нашем случае роль адсорбата играли Со или Mn. Следует отметить, что в случае повышения температуры осаждения адсорбата смещения точки Дирака не наблюдалось. Дальнейшее нанесение кобальта привело к появлению энергетической щели в спектре дираковских поверхностных состояний. Существование энергетической щели было зафиксировано при покрытиях от $0.4 \AA$ и более, по крайней мере до $2 \AA$, что примерно соответствует постоянной решетки кобальта $(2.5 \AA)$. Для детального показа энергетической щели в работе были рассчитаны вторые производные $d^{2} I / d E^{2}$ от изображения и проведены профили распределения энергии при различных значениях $k_{\|} \mathrm{c}$ использованием профилей Войта. На рис. 1 показаны спектры ЛФЭСУР топологических поверхностных состояний $\mathrm{BiSbTeSe}_{2}$ вблизи точки Дирака для образцов с $0.1(a), 0.4(b)$ и $0.6 \AA$ Со, в сопоставлении с соответствующими вторыми производными $d^{2} I / d E^{2}$ соответственно. Энергетическая щель вблизи точки Дирака хорошо заметна. Профили распределения интенсивности, соответствующие $0.6 \AA$ Со, показаны на рис. $1, g$. Данные на этом рисунке были подогнаны с использованием двух профилей Войта для нижнего и 

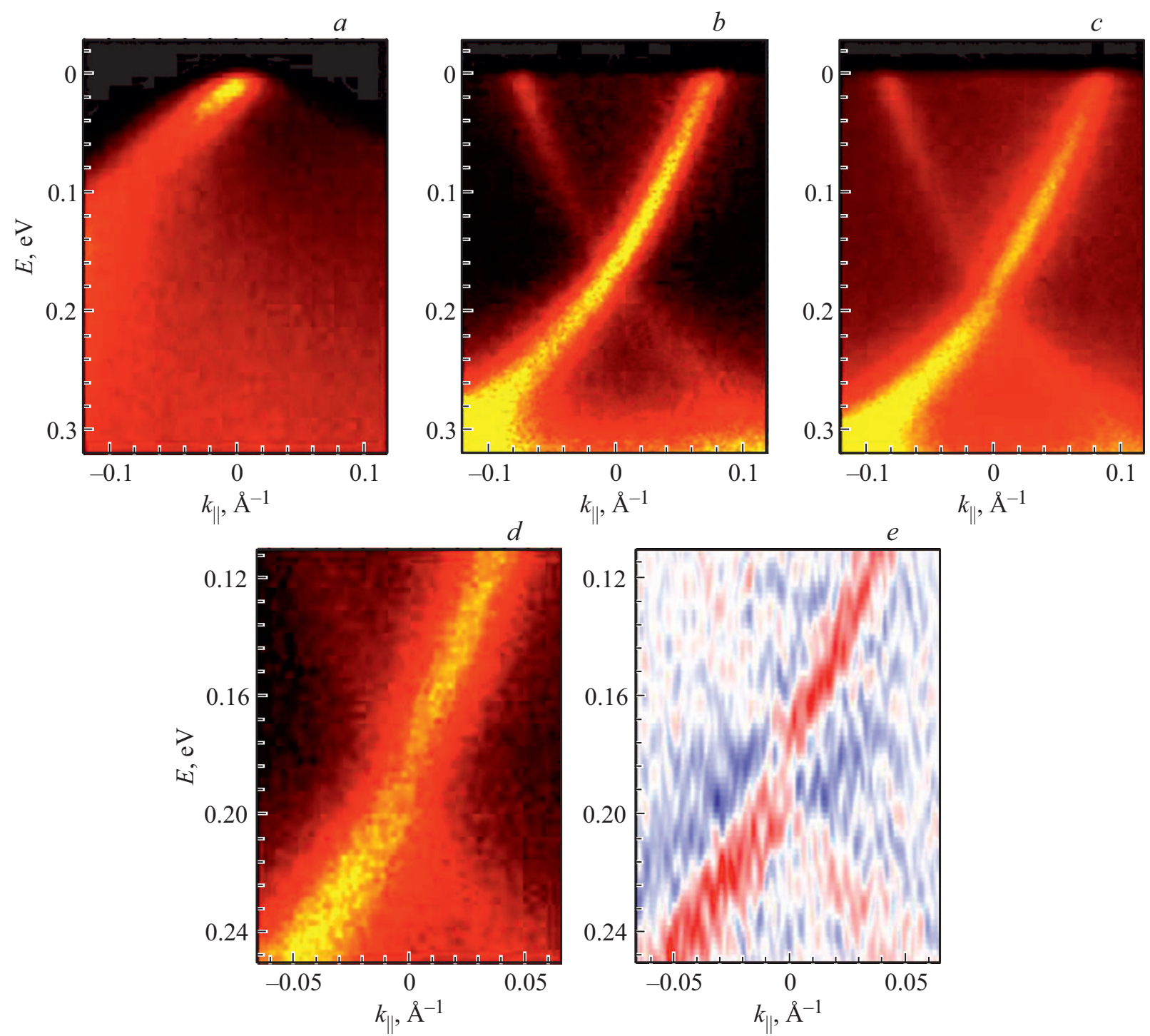

Рис. 3. $a-$ электронная структура поверхности $\operatorname{BiSbTeSe}_{2}$, измеренная методом ЛФЭСУР $(h v=6.3$ эВ, $T=20 \mathrm{~K})$ после скалывания образца в вакууме, $b-$ осаждение $0.1 \AA \mathrm{Mn}$ при комнатной температуре, $c-$ последующее осаждение $0.4 \AA$ Со при $300^{\circ}$ C. $d-$ увеличенное изображение области точки Дирака $(c)$ и его второй производной $d^{2} I / d E^{2}(e)$.

верхнего состояний конуса Дирака и с учетом фона Ширли. Третий профиль Войта был использован для учета асимметрии верхнего конуса Дирака. Полученные энергетические положения установленных пиков также показаны на рис. 1,c,e. Ширина энергетической щели может быть оценена как $21 \pm 6$ мэВ, независимо от величины покрытия кобальтом.

Раскрытие энергетической щели было зафиксировано для покрытий Со от $0.4 \AA$ и более, по крайней мере до $2 \AA$ (см. рис. 2, спектры для различного количества Со), что приблизительно равно постоянной решетки кобальта $(2.5 \AA)$. Из рисунков видно, что ширина щели оказалась постоянной, не зависящей от количества осажденного кобальта. Кроме того, было показано, что наличие энергетической щели при высокотемпературном осаждении Со не зависит от состава адсорбата, предварительно осажденного при комнатной температуре. На рис. $3, a-d$ показаны спектры ЛФЭСУР для покрытия $0.4 \AA \mathrm{Co}$, нанесенного при комнатной температуре с предварительно нанесенным $0.1 \AA$ адсорбата $\mathrm{Mn}$. Таким образом, энергетическая щель наблюдалась как в случае использования Со, так и $\mathrm{Mn}$ в качестве адсорбата.

Предварительные результаты моделирования показали, что при нанесении металла последний замещает атомы сурьмы и висмута во втором слое квинтислоя. При этом морфология поверхности не меняется. При достижении некоторого критического количества начинается образование островков новой фазы. Для кобальта это $\mathrm{CoTe}_{x} \mathrm{Se}_{2-x}$ [10]. Щель в спектре поверхностных состояний соответствует участкам замещенного пниктогена. Увеличение количества островков новой фазы приводит к размытию спектров ЛФЭСУР за счет уменьшения эффективной площади поверхности, связанной с раскрытием щели. 
Были проведены измерения зависимости ширины щели от температуры в диапазоне $15-150 \mathrm{~K}$, при этом какого-либо изменения ширины щели не было замечено. Щели магнитной и немагнитной природы были обнаружены, соответственно, в работах $[11,12]$ : при изучении объемного ТИ $\mathrm{Bi}_{2} \mathrm{Se}_{3}$, легированного $\mathrm{Mn}$, - в первой работе, и при интеркаляции ионов $\mathrm{Mn}$ в $\mathrm{Bi}_{2} \mathrm{Se}_{3}$ в процессе МЛЭ - во второй работе. Существование щели, имеющей немагнитную природу, может быть связано с гибридизацией поверхностных топологических состояний от двух взаимно противоположных поверхностей топологического изолятора, возникающих при диффузии атомов металла в ван-дер-ваальсовы зазоры между квинтислоями топологических изоляторов на базе халькогенидов и разрушающих ван-дер-ваальсовую связь на глубине от 6 квинтислоев [13], что приводит к „отщеплению“ этих слоев и образованию тонкой пленки ТИ, в которой влияние объемных состояний мало. Однако в рассматриваемом нами случае данный механизм неприменим из-за сверхмалого количества осажденного вещества $(0.4-2 \AA)$, не достаточного для диффузии через 6 квинтислоев подложки. Таким образом, наблюдаемая щель имеет, по-видимому, магнитную природу. Раскрытие энергетической щели магнитной природы можно рассматривать в качестве способа управления топологическими состояниями топологического изолятора.

\section{4. Заключение}

В работе было обнаружено, что при осаждении Со на поверхность $\mathrm{BiSbTeSe}_{2}(0001)$ с эффективной толщиной покрытия 0.4-2 $\AA$ происходит открытие энергетической щели вблизи точки Дирака. Ширина щели составляет $21 \pm 6$ мэВ. Щель имеет, предположительно, магнитную природу, связанную с поверхностным магнетизмом, так как наблюдается при сверхмалых количествах ферромагнитного адсорбата, не достаточных для проникновения в глубокие ван-дер-ваальсовы зазоры. Следует отметить, что существует крайне небольшое количество работ, посвященных экспериментальному наблюдению систем $\mathrm{Me} /$ ТИ с открытой энергетической щелью вблизи точки Дирака. Наблюдаемое в этих работах раскрытие щели (как правило, с использованием атомов $\mathrm{Mn}$ ) происходит при легировании ТI ферромагнитным металлом. В то же время отсутствуют работы, связанные с открытием энергетической щели в системе Me/TI при осаждении сверхмалых количеств ферромагнитного металла на поверхность ТІ. В частности, предыдущие попытки открыть энергетическую щель путем осаждения $\mathrm{Fe}$ и $\mathrm{Co}$ на поверхности $\mathrm{Bi}_{2} \mathrm{Se}_{3}$ и $\mathrm{Bi}_{2} \mathrm{Te}_{3}$ при комнатной температуре были безуспешными [14-16]. Также следует отметить, что раскрытие щели при нанесении немагнитных адсорбатов невозможно в силу самой природы топологически защищенных поверхностных состояний.

\section{Финансирование}

Работа поддержана грантом Российского фонда фундаментальных исследований № 17-02-00729.

\section{Конфликт интересов}

Авторы заявляют, что у них нет конфликта интересов.

\section{Список литературы}

[1] T.H. Hsieh, H. Lin, J. Liu, W. Duan, A. Bansil, L. Fu. Nature Commun., 3, 982 (2012).

[2] M.Z. Hasan, C.L. Kane. Rev. Mod. Phys., 82, 3045 (2010).

[3] X.-L. Qi, T.L. Hughes, S.-C. Zhang. Phys. Rev. B, 78, 195424 (2008).

[4] C. Kane. Nature Phys., 4 (5), 348 (2008).

[5] A.M. Shikin, A.A. Rybkina, D.A. Estyunin, D.M. Sostina, I.I. Klimovskikh, V.Yu. Voroshnin, A.G. Rybkin, K.A. Kokh, O.E. Tereshchenko, L. Petaccia, G.Di Santo, A. Kimura, P.N. Skirdkov, K.A. Zvezdin, A.K. Zvezdin. Sci. Rept., 8, 6544 (2018).

[6] L.A. Walsh, C.M. Smyth, A.T. Barton, Q. Wang, Z. Che, R. Yue, Ji. Kim, M.J. Kim, R.M. Wallace, C.L. Hinkle. J. Phys. Chem. C, 121 (42), 23551 (2017).

[7] E. Longo, C. Wiemer, R. Cecchini, M. Longo, A. Lamperti, A. Khanas, A. Zenkevich, M. Fanciulli, R. Mantovan. J. Magn. Magn. Mater., 474, 632 (2019).

[8] A.K. Kaveev, N.S. Sokolov, Se.M. Suturin, N.S. Zhiltsov, V.A. Golyashov, K.A. Kokh, I.P. Prosvirin, O.E. Tereshchenko, M. Sawada. Cryst. Eng. Commun., 20, 3419 (2018).

[9] H. Iwasawa, E.F. Schwier, M. Arita, A. Ino, H. Namatame, M. Taniguchi, Y. Aiura, K. Shimada. Ultramicroscopy, 182, 85 (2017).

[10] A.K. Kaveev, S.M. Suturin, V.A. Golyashov, K.A. Kokh, O.E. Tereshchenko. J. Phys.: Conf. Ser., 055016, 1400 (2019).

[11] Y.L. Chen, J.-H. Chu, J.G. Analytis, Z.K. Liu, K. Igarashi, H.-H. Kuo, X.L. Qi, S.K. Mo, R.G. Moore, D.H. Lu, M. Hashimoto, T. Sasagawa, S.C. Zhang, I.R. Fisher, Z. Hussain, Z.X. Shen. Science, 329, 5992, 659 (2010).

[12] J. Sanchez-Barriga, A. Varykhalov, G. Springholz, H. Steiner, R. Kirchschlager, G. Bauer, O. Caha, E. Schierle, E. Weschke, A.A. Ünal, S. Valencia, M. Dunst, J. Braun, H. Ebert, J. Minár, E. Golias, L.V. Yashina, A. Ney, V. Holý, O. Rader. Nature Commun., 7, 10559, (2016).

[13] Y. Zhang, K. He, C.-Z. Chang, C.-L. Song, L.-L. Wang, X. Chen, J.-F. Jia, Zh. Fang, X. Dai,W.-Y. Shan, Sh.-Q. Shen, Q. Niu, X.-L. Qi, Sh.-Ch. Zhang, X.-C. Ma, Q.-K. Xue. Nature Physics, 6, 584 (2010).

[14] M. Ye, S.V. Eremeev, K. Kuroda, E.E. Krasovskii, E.V. Chulkov, Y. Takeda, Y. Saitoh, K. Okamoto, S.Y. Zhu, K. Miyamoto, M. Arita, M. Nakatake, T. Okuda, Y. Ueda, K. Shimada, H. Namatame, M. Taniguchi, A. Kimura. Phys. Rev. B, 85, 205317 (2012).

[15] M.R. Scholz, J. Sáchez-Barriga, D. Marchenko, A. Varykhalov, A. Volykhov, L.V. Yashina, O. Rader. Phys. Status Solidi RRL, 7, 139 (2013).

[16] M.R. Scholz, J. Sánchez-Barriga, D. Marchenko, A. Varykhalov, A. Volykhov, L.V. Yashina, O. Rader. Phys. Rev. Lett., 108, 256810 (2012).

Редактор А.Н. Смирнов 


\section{Band gap opening at the Dirac point after the Co deposition on the (0001) surface of $\mathrm{BiSbTeSe}_{2}$ topological insulator}

A.K. Kaveev ${ }^{1}$, A.G. Banshchikov ${ }^{1}$, A.N. Terpitskiy ${ }^{1}$, V.A. Golyashov' ${ }^{2}$ O.E. Tereshchenko ${ }^{2}, K . A . K^{\prime} k^{3}{ }^{3}$, D.A. Estyunin ${ }^{4}$, A.M. Shikin ${ }^{4}$

${ }^{1}$ loffe Institute, 194021 St. Petersburg, Russia

${ }^{2}$ Rzhanov Institute of Semiconductor Physics, 630090 Novosibirsk, Russia

${ }^{3}$ Sobolev Institute of geology and mineralogy, 630090 Novosibirsk, Russia

${ }^{4}$ Novosibirsk State University, 199034 St. Petersburg, Russia

Abstract It was shown for the first time that Co subnanometer coaverage, being deposited by molecular beam epitaxy method onto the (0001) surface of the $\mathrm{BiSbTeSe}_{2}$ topological insulator at $330^{\circ} \mathrm{C}$, opens an energy band gap in the spectrum of topological surface states in the region of the Dirac point, with a shift in the position of the Dirac point caused by preliminary deposition of the adsorbate at room temperature. The gap band width is $21 \pm 6 \mathrm{meV}$. Temperature-dependent measurements in the $15-150 \mathrm{~K}$ range did not show any width changes. 